Sādhanā Vol. 40, Part 3, May 2015, pp. 973-983. (C) Indian Academy of Sciences

\title{
Effect of ultra-fast mixing in a microchannel due to a soft wall on the room temperature synthesis of gold nanoparticles
}

\author{
M K S VERMA and V KUMARAN* \\ Department of Chemical Engineering, Indian Institute of Science, Bangalore \\ 560012, India \\ e-mail: kumaran@chemeng.iisc.ernet.in
}

MS received 29 September 2014; revised 4 February 2015; accepted 6 February 2015

\begin{abstract}
The room-temperature synthesis of mono-dispersed gold nanoparticles, by the reduction of chlorauric acid $\left(\mathrm{HAuCl}_{4}\right)$ with tannic acid as the reducing and stabilizing agent, is carried out in a microchannel. The microchannel is fabricated with one soft wall, so that there is a spontaneous transition to turbulence, and thereby enhanced mixing, when the flow Reynolds number increases beyond a critical value. The objective of the study is to examine whether the nanoparticle size and polydispersity can be modified by enhancing the mixing in the microchannel device. The flow rates are varied in order to study nanoparticle formation both in laminar flow and in the chaotic flow after transition, and the molar ratio of the chlorauric acid to tannic acid is also varied to study the effect of molar ratio on nanoparticle size. The formation of gold nanoparticles is examined by UV-visual spectroscopy and the size distribution is determined using scanning electron microscopy. The synthesized nanoparticles size decreases from $\geq 6 \mathrm{~nm}$ to $\leq 4 \mathrm{~nm}$ when the molar ratio of chlorauric acid to tannic acid is increased from 1 to 20 . It is found that there is no systematic variation of nanoparticle size with flow velocity, and the nanoparticle size is not altered when the flow changes from laminar to turbulent. However, the standard deviation of the size distribution decreases by about $30 \%$ after transition, indicating that the enhanced mixing results in uniformity of particle size.
\end{abstract}

Keywords. Nanoparticle; instability; microfluidics; microfluidic mixing; transition to turbulence.

\section{Introduction}

Micro-fluidic devices have attracted great interest due to their potential for miniaturization of chemical synthesis, separation, polymer synthesis, bio-reagent analysis and drug delivery. Miniaturization results in fast response, lower sample and reagent consumption, lower cost, higher

${ }^{*}$ For correspondence 
degree of integration and precision, and potential for synthesis on demand at point of delivery. In parallel, the synthesis of mono-disperse nanoparticles of size below $10 \mathrm{~nm}$ has also attracted considerable scientific and commercial interest, since the particles have unique chemical, electronic, magnetic, optical properties which are of use in the fields like catalysis (Somorjai \& Park 2008), electronics (Markovich et al 1997), sensors (Shipway et al 2000), drug delivery (Panyam \& Labhasetwar 2003), bio-sensing and bio-imaging (Boisselier \& Astruc 2009), medicine (Giljohann et al 2010) and cancer treatment (Peng et al 2009). Gold nanoparticles, the subject of the present work, have been of interest in nanoelectronics applications (Schmid \& Simon 2005; Schmid 2008) as well as carriers in drug delivery. For all these applications, the size and size distribution of nanoparticles are significant because of their size dependent properties. In nanoelectronics applications, accurate size control is required to fabricate regular large-area arrays, which can then be used in a variety of applications including high-density memory devices and for growth of regular nano-wire arrays for dye-sensitized solar cells. In diagnostics and drug delivery, gold nanoparticles are often used as carriers for drug molecules, since they can be complexed with thiol groups attached to the drug molecules. Their small size enables preferential transport through pores and thereby provides targeted drug delivery. However, efficient renal clearance requires the hydrodynamic radius of the drug complex to be less than $20 \mathrm{~nm}$, and this places a restriction of about $5 \mathrm{~nm}$ on the size of the nanoparticle carrier (Choi et al 2010). Hence, controlling the physical dimension and size distribution of such particles is one of the important issues in the synthesis of nanoparticles.

The conventional method of nanoparticle synthesis includes gas-phase synthesis and solutionbased synthesis. In gas phase techniques, nanoparticle are synthesized by laser evaporation (Perriere et al 2007), sputtering, flame atomization and flame spray pyrolysis (FASP) (Teoh et al 2010; Rudin et al 2011) and plasma-based nanoparticle synthesis (Matsui 2006). The solution-based techniques involve the chemical reduction of metal salt in aqueous, organic phase or aqueous/organic two phase systems. For example, in Brust method (Brust et al 1994), gold chloride $\left(\mathrm{AuCl}_{4}\right)$ is transferred from aqueous phase to organic phase (toluene) using tetraoctylammonium bromide and reduced with aqueous sodium borohydride in the presence of alkylthiols. In aqueous phase synthesis, nanoparticles are synthesized from chemical reduction of $\mathrm{Au} \mathrm{Cl}_{4}$ by reducing agents such as tannic acid (Sivaraman et al 2010), citric acid and ascorbic acid (Turkevich et al 1951; Kimling et al 2006). Usually, these synthesis methods are carried out in batch, and the size and size distribution of the nanoparticles are adversely affected by inhomogeneities inherent in batch operations. Recently, microfluidic devices have been used for nanoparticle synthesis (Song et al 2004; Li et al 2008; Hung \& Lee 2007; Wagner et al 2004; Jahn et al 2008; Rhee et al 2011; Lee et al 2009; Karnik et al 2008; Wang et al 2010).

These syntheses at the micro-scale can be better controlled in comparison to conventional methods, and there is the possibility of fast reaction, less consumption of expensive reagents and better control over size distribution. An additional advantage over bulk synthesis methods is that particles can be synthesized according to demand at the point of delivery. However, the size and quality of nanoparticles synthesized is sensitive to the efficiency of rapid mixing of reagents. Rapid and efficient mixing at micro-scale is a challenging problem because the flow is laminar at low Reynolds number, and mixing occurs due to molecular diffusion. The time required for the mixing of two fluids across a distance $h$ can be estimated as $\left(h^{2} / D\right)$, where $\mathrm{D}$ is the diffusion coefficient (Ottino and Wiggins 2004). For liquids, the diffusion coefficient (Ottino \& Wiggins 2004) of small molecules is about $10^{-9} \mathrm{~m}^{2} / \mathrm{s}$, while that for complex and polymeric molecules can be as low as $10^{-13} \mathrm{~m}^{2} / \mathrm{s}$. This results in a diffusion time of between $10^{3} \mathrm{~s}$ and $10^{7} \mathrm{~s}$ for a microchannel of width $1 \mathrm{~mm}$. Clearly, these mixing times are too long for mixing in practical applications, and it is necessary to induce mixing by artificial means. 
A number of techniques have been developed in order to increase the efficiency of mixing (Capretto et al 2011; Verma et al 2008; Hessel et al 2005; Lee et al 2011). These techniques can be broadly classified as active and passive mixing. In active mixing, an external force is used to generate secondary flows and thereby accelerate the mixing process. Several strategies such as acoustic mixing due to sound waves, mixing by micro-rotors and thermal actuation have been explored (Kane et al 2008; Jiang et al 2004; Liu et al 2000; Stroock et al 2002; Knight et al 1998; Bessoth et al 1999; Lee et al 2006; Glasgow \& Aubry 2003; Posner \& Santiago 2006). The disadvantage of these is the additional input of energy and the requirement or expensive machining of small parts. In passive micro-mixing, there are no moving parts, but the fluid streamlines are stretched and folded in a periodic manner along the channel in order to achieve better contact between the two fluid streams. Strategies include tortuous flow pathways in order to mix the fluid streamlines, generation of chaotic advection due to grooves on the wall of the channel, periodic split-and-rejoin strategies both in series and parallel in order to mix the fluid streams. These strategies have the advantage that external energy input is not required, while the main disadvantages are the significantly higher pressure gradient to drive the flow due to higher fluid friction in the tortuous paths, and the micro-machining of small parts. Another strategy that has been used is to induce an electrokinetic instability (Lin et al 2004) due to the application of an electric field, which influences the motion of ions within the fluid. This strategy requires dissolved ions in the fluids in order to generate the instability, and so it is not useful for organic fluids.

A recent advance in ultra-fast microfluidic mixing due to a dynamical instability induced by a soft wall is reported by Verma \& Kumaran $(2012,2013)$, where the dynamical coupling between the fluid and the wall dynamics results in a spontaneous flow instability which generates mixing comparable to that in turbulent flows. The transition Reynolds number for the flow instability is significantly lower than the critical Reynolds number for the flow through rigid-walled channels. The transition flow rate can be tuned by changing wall properties, because the transition Reynolds number decreases as the shear modulus of the wall decreases. It has been shown that the mixing time is up to five orders of magnitude lower than that due to molecular diffusion. Here, this 'soft mixing' is used for synthesis of gold nanoparticles in a microchannel, and the effect of mixing on the size and polydispersity of the nanoparticles is examined.

\section{Experimental methods}

\subsection{Micro-channel geometry}

A split inlet micro-channel geometry is used for mixing experiments as shown in figure 1 . The channel is $100 \mu \mathrm{m}$ deep $(\mathrm{H}), 1.5 \mathrm{~mm}$ in width $(\mathrm{W})$ and $\sim 4 \mathrm{~cm}$ in length $\left(\mathrm{L}_{\mathrm{d}}+\mathrm{L}_{\mathrm{m}}\right)$.

There is a 'development section' of length $\left(\mathrm{L}_{\mathrm{d}}=0.8 \mathrm{~cm}\right)$ from the inlet for which all the walls of channel are made with hard gel to reduce disturbances at the inlet to the test section. For 'test

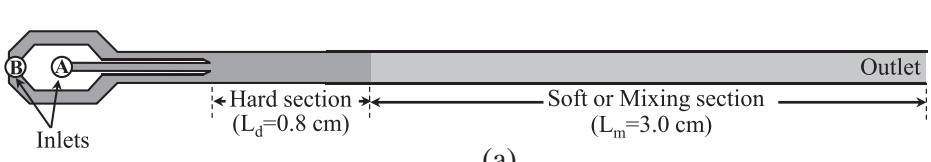

(a)

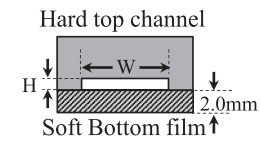

(b)

Figure 1. Schematic of the micro-channel configuration with split inlet (a) and cross-section of the channel in soft/mixing section (b). 




Figure 2. Experimental setup for preparation of gold nanoparticles.

section' of length $\left(\mathrm{L}_{\mathrm{m}}=3.0 \mathrm{~cm}\right)$, the bottom wall is made with low shear modulus. There are two fluid inlets, which enables us to inject two different fluid streams at different flow rates. The flow rate ratio of inlet $\mathrm{A}$ to $\mathrm{B}$ was maintained to 1:5 throughout all the experiments, so that there is no shearing between the two streams.

Micro-channels were fabricated with poly-dimethylsiloxane (PDMS) polymer using soft lithography techniques, as discussed in Verma \& Kumaran (2013). The micro-channels have three rigid walls made of hard gel, and one soft wall made with a lower catalyst concentration to obtain a low shear modulus. The catalyst concentration was varied in order to alter the shear modulus of the soft wall. The hard and soft sections are made separately, and then bonded together (Satyanarayana et al 2005), to form a rectangular microchannel of width $1.5 \mathrm{~mm}$ and height $100 \mu \mathrm{m}$.

The nanoparticle synthesis setup consists of flexible microchannel device, channel holder, glass vial, syringe pumps, syringes and silicone tubing as depicted in figure 2 . Two syringe pumps (A and B in figure 2) were used to pump the Chloroauric acid solution and tannic acid into the microchannel. The microchannel sample was fixed to the channel holder vertically to ensure easy and clean sample collection. All the glassware, syringes and silicone tubing were cleaned thoroughly with aqua regia (mixture of nitric acid and hydrochloric acid in a volume ratio of 1:3) and rinsed with de-ionized water before synthesis. In order to study the effect of flow rate on particle size and distribution, the Reynolds number was defined based on the flow rate $\left(Q=Q_{\mathrm{A}}+Q_{\mathrm{B}}\right)$ and the width of the channel as $R e=\frac{\rho Q}{W \eta}$, and residence time was defined as $R_{t}=\frac{L_{m} W H}{Q}$. Here, $W$ is the width of the channel, $\rho$ and $\eta$ are fluid density and viscosity respectively. The soft wall was made of PDMS gel with a low shear modulus of two different values, $18 \mathrm{kPa}$ and $25 \mathrm{kPa}$ (in contrast to the shear modulus of $0.55 \mathrm{mPa}$ for hard PDMS gel fabricated according to the standard protocol). For shear modulus $18 \mathrm{kPa}$, the transition takes place at a Reynolds number of about 200, and for shear modulus $25 \mathrm{kPa}$, the transition takes place at a Reynolds number of about 289. The transition is illustrated in figure 3 for a soft wall with shear modulus $18 \mathrm{kPa}$. Here, clear water is pumped into inlet $\mathrm{B}$, and black dye is pumped through inlet $\mathrm{A}$ in figure 1 , and the progress of the dye stream is tracked using high speed imaging from above. When the Reynolds number is below the transition Reynolds number, the flow is laminar

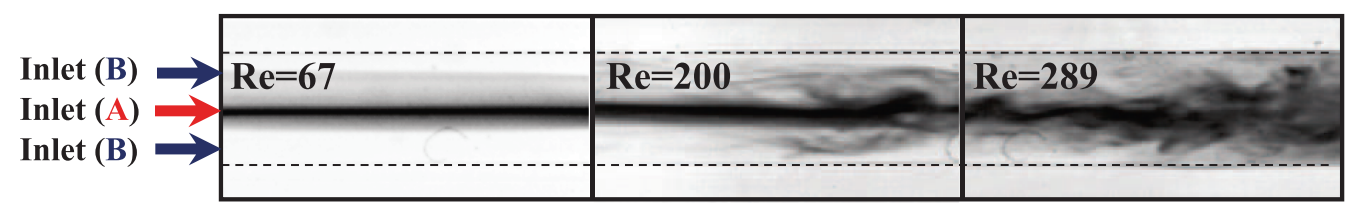

Figure 3. Dye-stream images showing the flow structure in micro-channel at different Reynolds numbers for soft wall with shear modulus $18 \mathrm{kPa}$ at locations $1.5 \mathrm{~cm}$ downstream. 
and the dye stream progresses in a smooth fashion. Above the transition Reynolds number, a catastrophic breakup of the dye stream is observed, and discussed in more detail in Verma \& Kumaran (2013).

Gold nanoparticles were synthesized by the reduction of chlorauric acid $\left(\mathrm{HAuCl}_{4}\right)$ with tannic acid as the reducing and stabilizing agent in the microchannel. Chloroauric acid (SigmaAldrich), tannic acid (Acros/Merck), potassium carbonate (Merck), hydrochloric acid (S.D.fine chemicals) and de-ionized water (DI) obtained from a MilliQ system were used in all the experiments. For variation in size of the nanoparticles, the molar ratio of tannic acid to chloroauric acid was varied. In a typical experiment for molar ratio of $2.0,1 \mathrm{ml}$ of $25.4 \mathrm{mM}$ chloroauric acid was dissolved in $16 \mathrm{ml}$ of DI water for the chlorauric acid solution. For the tannic acid solution, $9 \mathrm{ml}$ of $5.88 \mathrm{mM}$ tannic acid was added to $72 \mathrm{ml}$ of DI water and $72.5 \mathrm{mM}$ potassium carbonate solution was used to adjust the $\mathrm{pH}$ of tannic acid solution to 7 . Both solutions were pumped into the channel using two syringe pumps. The molar ratio of the two reactants was altered by changing the concentrations of the chlorauric acid and tannic acid while keeping the flow rates the same. This mixing of streams leads to the generation of gold nanoparticles by reduction of $\mathrm{HAuCl}_{4}$ by tannic acid. The synthesized nanoparticles were collected at outlet of the channel in glass vials as depicted in figure 2. In order to compare the formation of the nanoparticles in the microchannel with those made in a batch process, a separate set of batch experiments is carried out. Here, the reactants in the desired molar ratio are mixed in a $10 \mathrm{ml}$ beaker and stirred well using a magnetic stirrer (TARSONS SPINOT-Digital Magnetic Stirrer Hot Plate) at $1000 \mathrm{rpm}$. The sample from the beaker is then used for determining the nanoparticle size and polydispersity.

\subsection{Characterization techniques}

An ultraviolet-visible (UV-Vis) spectrophotometer (PG Instruments) was used to determine the surface plasmon resonance (SPR) for metallic gold nanoparticles. The Ultraviolet-Visible absorption spectra were recorded in the range of 400-600 $\mathrm{nm}$ within $1 \mathrm{~h}$ of synthesis of nanoparticles. Here, DI water was used as reference. The evolution of the nanoparticle concentration and size can also be determined using the UV-Visible absorption spectroscopy. In this procedure, the output from the microchannel was immediately transferred to the cuvette and the absorption spectra were recorded for $30 \mathrm{~s}$. The absorption spectra were then again recorded after $15 \mathrm{~s}$ in order to determine the time take for reaching the final nanoparticle size.

Field Emission Scanning Electron Microscopy (FESEM) was performed for particle size analysis. The specimens for imaging were prepared by evaporation of one or two drops of chloroform solution of the nanoparticles on a silicon wafer $(2 \mathrm{~mm} \times 2 \mathrm{~mm})$. Prior to use, the silicon wafer was cleaned by ultrasonicating in toluene, followed by acetone and ethanol for 5 min each. For drop casting the nanoparticle on silicon wafer, it is essential to transfer nanoparticles from an aqueous phases to organic phases such as chloroform. This phase transfer of nanoparticles was achieved by capping the nanoparticles with Dodecanethiol (1-Dodecylmercaptane, Aldrich). In a typical experiment, $5 \mathrm{ml}$ of colloidal gold solution was mixed with $5 \mathrm{ml}$ of $1 \mathrm{mM}$ solution of dodecanethiol in ethanol, and one drop of hydrochloric acid. The mixture was left for $3 \mathrm{~h}$ and then centrifuged and washed twice with ethanol and once in acetone. The precipitated nanoparticle was left for $24 \mathrm{~h}$ for drying. The dried nanoparticles were dispersed in $5 \mathrm{ml}$ of chloroform and used for drop-casting on silicon wafer. The FESEM images were processed using ImageJ software and MATLAB for analyzing particle size and distribution as shown in figure 4. In all our results, at least 500 particles were considered for obtaining the size distribution, the mean diameter and the standard deviation. In addition to obtaining the mean and standard deviation over the entire sample, the sample is also divided into four equal groups, and the mean and 

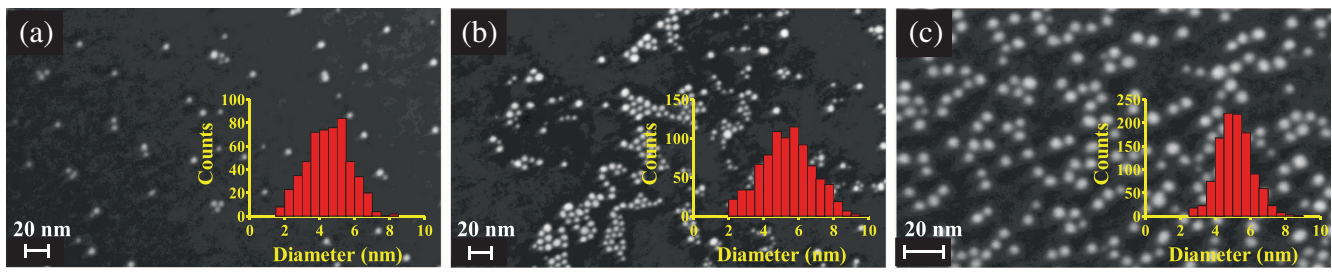

Figure 4. FESEM image of nanoparticles prepared in microchannel with shear modulus $18 \mathrm{kPa}$ at $\operatorname{Re}=0$ (a); $\operatorname{Re}=156$ (b); $\operatorname{Re}=289$ (c). Insets show size distribution histogram.

standard deviation for each of these groups are calculated separately. The variance in the mean and standard deviation over the four groups are used to plot the error bars in the mean and standard deviation distributions for each sample.

Transmission Electron Microscopy (TEM) imaging was performed using a Tecnai T20 operating at $200 \mathrm{KV}$ for size analysis and diffraction (SAED) pattern of gold nanoparticles. The specimens for TEM were prepared by drop casting and ImageJ software was used for image analysis.

\section{Experimental results}

The influence of ultra-fast mixing on the nanoparticle size and distribution was investigated. At the same time, the effect of molar ratio of tannic acid to chloroauric acid was also investigated and discussed. As discussed earlier, ultra-fast mixing was achieved by making one channel wall with low shear modulus ( $18 \mathrm{kPa}$ and $25 \mathrm{kPa}$ ), such that there is a spontaneous instability of the laminar flow when the Reynolds number exceeds a critical value. Figure 3 shows that the laminar to turbulent transition takes place, when Reynolds number exceeds critical value (Re $=200$ for shear modulus $18 \mathrm{kPa}$ ). The physical mechanism of this transition is a dynamical instability due to the fluid-wall coupling which results in a transition from the laminar flow to a more complicated profile, resulting in the catastrophic break-up of streamlines in the flow. This catastrophic break-up of streamlines results in ultra-fast mixing in the microchannel.

Figure 5 shows the transmission electron microscopy (TEM) image of gold nanoparticles synthesized at different Reynolds numbers in microchannel device for molar ratio $(\mathrm{MR}=3)$. Figure 5(a) shows the TEM image of non-spherical nanoparticles synthesized in batch mode, which we label as $\mathrm{Re}=0$ for the present purposes. The nanoparticles synthesized in microchannel after transition Reynolds number are shown in figure 5(b) and (c). We can clearly see much
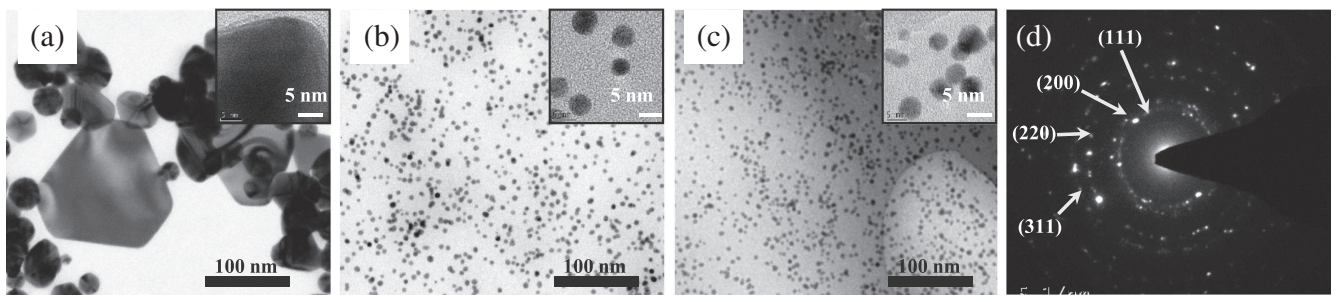

Figure 5. TEM image of nanoparticles prepared in batch mode $\mathrm{Re}=0$ (a); TEM image of nanoparticles prepared in microchannel at $\operatorname{Re}=289$ (b); at $\operatorname{Re}=467$ (c). The inset shows higher resolution images. The diffraction pattern of gold nanoparticles (d). Insets show higher magnification images. 


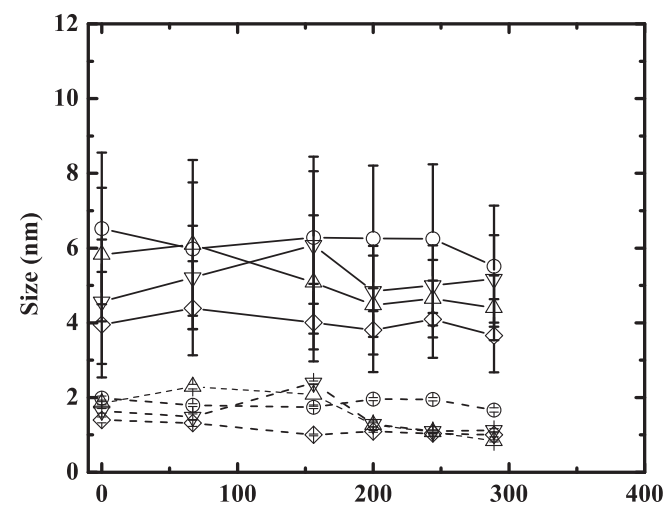

(a)

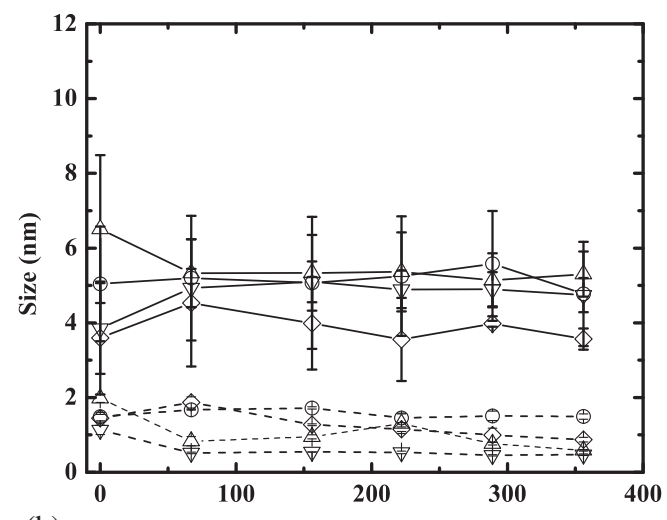

(b)

Re

Figure 6. The mean (solid lines) and standard deviation (dashed lines) of the nanoparticle size distribution as a function of the Reynolds number for a straight channel; (a) micro-channel with shear modulus $18 \mathrm{kPa}$, in which the molar ratio is $1(\circ), 3(\triangle), 4(\nabla)$ and $20(\diamond)$; (b) micro-channel with shear modulus $25 \mathrm{kPa}$, in which the molar ratio is $2(\circ), 3(\triangle), 4(\nabla)$ and $9(\diamond)$. Here, $\operatorname{Re}=0$ corresponds to nanoparticles prepared in batch mode.

smaller $(\leq 5 \mathrm{~nm})$ and uniform particles prepared after transition Reynolds number. The ultra-fast mixing in microchannel facilitates the formation of smaller mono-dispersed nanoparticle seeds, which results in uniform particle growth and decreased polydispersity. Figure 5(d) shows the diffraction pattern with rings corresponding to the (111), (200), (220), and (311) revealing the face-centered-cubic (fcc) structure of gold nanoparticle. The diffraction (SAED) pattern also shows the particles are crystalline in the direction of a singular axis and not polycrystalline in nature.


Figure 7. UV-Visible spectra of gold nanoparticles for different molar ratios (a); the time-lapse UVVisible spectra of gold nanoparticle solutions for molar ratio $(M R=3)(b)$. The dashed lines show the spectra for the nanoparticles prepared in batch mode, the solid and dotted lines show the spectra for the nanoparticles prepared in microchannel with shear modulus $18 \mathrm{kPa}$, at Reynolds number $\mathrm{Re}=67$ $\left(R_{\mathrm{t}}=0.045 \mathrm{~s}\right)$ and $\mathrm{Re}=289\left(R_{\mathrm{t}}=0.011 \mathrm{~s}\right)$ respectively. Different colors showing UV-Visible spectra at different time. 
The molar ratio of tannic acid to chloroauric acid was also varied in order to study the effect on particle size and distribution. It was observed that bigger particles $(\geq 5 \mathrm{~nm}$ ) were formed for lower molar ratio $(\mathrm{MR}=1)$ and smaller particles $(\leq 5 \mathrm{~nm})$ for higher molar ratio $(\mathrm{MR} \geq 1)$ as shown in figure 6(a) and (b). But no effect of molar ratio on polydispersity was seen. The nanoparticle size distributions obtained for the synthesis in a microchannel are also shown in figure 6. The mean and standard deviation of the size distribution are shown as a function of Reynolds number in figure 6(a) for experiments in which the soft wall is made of gel with shear modulus $18 \mathrm{kPa}$, and in figure 6(b) for experiments in which the soft wall is made of gel with shear modulus $25 \mathrm{kPa}$. We see that there is a decrease in the polydispersity at the transition Reynolds number when the molar ratio is between 3 and 4, indicating that efficient mixing results in a smaller size distribution. The decrease is not significant at both the largest molar ratio of 20 where the polydispersity is already very small, or at smallest molar ratio of 1 where the polydispersity continues to be large possibly due to the formation of a large number of seeds which grow in different environments. However, at intermediate molar ratios, there is a clear decrease in the polydispersity due to the enhanced mixing.

The presence of gold nanoparticles was also confirmed by the UV-Visible spectroscopy. The UV-Vis spectra of gold nanoparticle solutions synthesized for molar ratio (1, 3, 4 and 20) at $\operatorname{Re}=289\left(R_{\mathrm{t}}=0.011 \mathrm{~s}\right)$ are shown in figure 7(a) for shear modulus $18 \mathrm{kPa}$. The surface plasmon resonance (SPR) peak in the UV-Vis spectra of gold nanoparticle formed at MR $=1$ shows positive shift from $520 \mathrm{~nm}$, indicating the presence of large particles, while particle formed at higher MR (3 and 4) shows negative shift, indicating the presence of smaller particles. We were not able to see any (SPR) peak in the UV-Vis spectra for MR 20 due to low particle concentration. The evolution of the UV-Visual spectrum with time was also recorded to study completion of the redox reaction inside the microchannel. The evolution of the UV-Visual spectrum was recorded as follows. The nanoparticle solutions from the output of the microchannel were immediately collected and transferred to the cuvette of the UV-Visual spectrometer, and scanned for $30 \mathrm{~s}$. After this, the sample was held for a waiting time of $15 \mathrm{~s}$ and then scanned again. This procedure

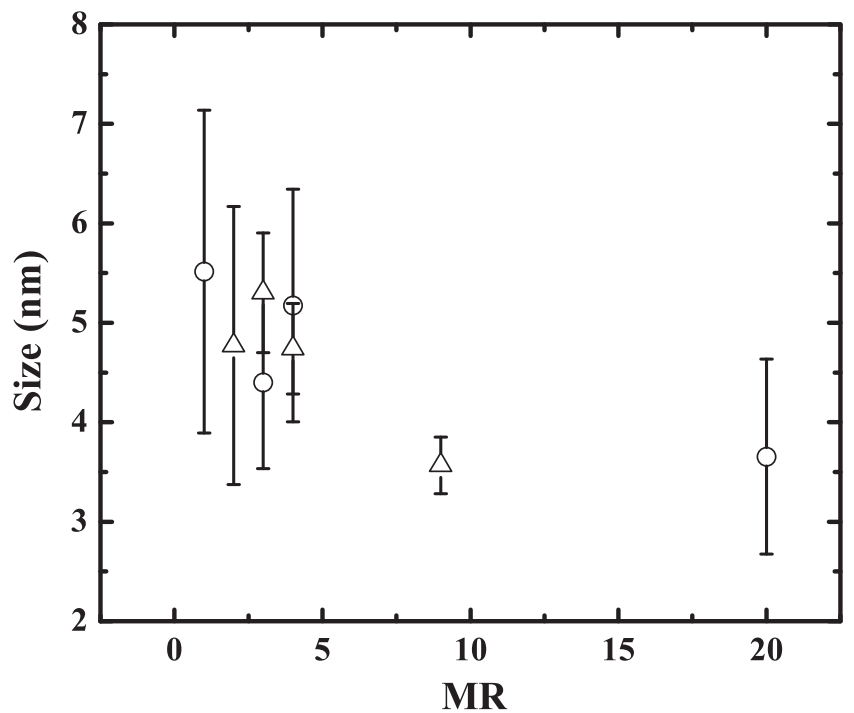

Figure 8. Nanoparticle size prepared at transition Reynolds number as a function of molar ratio; (o) microchannel with shear modulus $18 \mathrm{kPa}$; $(\triangle)$ microchannel with shear modulus $25 \mathrm{kPa}$. 
was repeated several time, in order to examine the evolution of the spectrum with time. The results are shown in figure 7(b). This figure shows that for nanoparticles prepared in the batch mode, there is a slow evolution of the spectrum with time, indicating particle ripening. However, for the nanoparticles prepared in the microchannel, there is a shift in the spectrum from the first scan to the second scan (a total waiting time of about $45 \mathrm{~s}$ ), but there is no further evolution in the spectrum with time. This indicates that the rapid mixing in microchannels significantly reduces the time required for ripening of the nanoparticles to their final size. The height of the peak also decreases as the Reynolds number is increased, indicating reduction in the nanoparticle size consistent with experimental observations. The nanoparticle size prepared at transition Reynolds number as a function of molar ratio is shown in figure 8 . We see a clear influence of molar ratio on nanoparticle size. Bigger particles of size $5.5 \mathrm{~nm}$ were formed at molar ratio (MR = $1)$, while those formed at MR value below $(\mathrm{MR}=1)$ shows systematic decrease in nanoparticle size.

\section{Conclusions}

The effect of ultra-fast mixing on the size and polydispersity of gold nanoparticles synthesized at room temperature reduction of chloroauric acid, with tannic acid as the reducing agent and stabilizer, has been studied. In conclusion, the present work demonstrates a significant advancement in terms of simple, rapid and continuous synthesis of mono-dispersed gold nanoparticles at room temperature. The average particle size does depend on molar ratio but does not depend on the mixing conditions in the channel, indicating that the average size is determined only by the chemistry. However, the polydispersity does vary with the mixing conditions. The polydispersity gets lower for high Reynolds number ( $\mathrm{Re}>200$ for shear modulus $18 \mathrm{kPa}$, channel). It is observed that polydispersity does not vary much with flow velocity, when the tannic acid is in excess compared to the chlorauric acid $(\mathrm{MR} \geq 3)$. The ultra-mixing of chlorauric acid with tannic acid results in the formation of large number of small nanoparticle seeds and uniform growth. This results in reduced polydispersity as compared to batch nanoparticle synthesis protocols. At an intermediate molar ratio $(M R \geq 3)$ of tannic acid to chlorauric acid, we obtain the lowest polydispersity, and the polydispersity does significantly decrease after transition. The rapid mixing in the microchannel also reduces the time required for ripening of the nanoparticles to their final steady size distribution. In contrast the ripening time in the batch experiments is a few minutes, we find that the final steady state is reached at less than $45 \mathrm{~s}$ after mixing in the microchannel, and there is no further evolution in the nanoparticle size after this. This indicates that mixing in the microchannel enables the rapid formation of nanoparticles. This could be useful for developing multi-step synthesis procedures in series or parallel in microreactors.

\section{Acknowledgements}

The authors would like to thank the Department of Science and Technology, Government of India, for financial support.

\section{References}

Bessoth F, De Mello A and Manz A 1999 Microstructure for efficient continuous flow mixing. Anal. Commun. 36: 213 
Boisselier E and Astruc D 2009 Gold nanoparticles in nanomedicine: Preparations, imaging, diagnostics, therapies and toxicity. Chem. Soc. Rev. 38: 1759-1782

Brust M, Walker M, Bethell D, Schiffrin D J and Whyman R 1994 Synthesis of thiol-derivatised gold nanoparticles. J. Chem. Soc. Chem. Commun. 801-802

Capretto L, Cheng W, Hill M and Zhang X 2011 Micromixing with in microfluidic devices. Microfluidics 304: 2768

Choi H S, Liu W, Liu F, Nasr K, Misra P, Bawendi M G and Frangioni J V 2010 Design considerations for tumor-targeted nanoparticles. Nat. Nanotechnol. 5: 4247

Giljohann D A, Seferos D S, Daniel W L, Massich M D, Patel P C and Mirkin C A 2010 Gold nanoparticles for biology and medicine. Angew Chem. Int. Ed. 49: 3280-3294

Glasgow I and Aubry N 2003 Enhancement of microfluidic mixing using time pulsing. Lab on a Chip 3: 114

Hessel V, Lowe H and Schonfeld F 2005 Micromixers - A review on passive and active mixing principles. Chem. Eng. Sci. 60: 24792501

Hung L H and Lee A P 2007 Microfluidic devices for the synthesis of nanoparticles and biomaterials. J. Med. Biol. Eng. 27(1): 1-6

Jahn A, Reiner J E, Vreeland W N, DeVoe D L, Locascio L E and Gaitan M 2008 Preparation of nanoparticles by continuous-flow microfluidics. J. Nanoparticle Res. 10(6): 925-934

Jiang F, Drese K S, Hardt S, Kupper M and Schonfeld F 2004 Helical flows and chaotic mixing in curved micro-channels. AIChE J. 50: 2297-2305

Kane Avinash S, Armin Hoffmann, Peter Baumgartel, Robert Seckler, Gerd Reichardt and Horsley David A 2008 Microfluidic mixers for the investigation of rapid protein folding kinetics using synchrotron radiation circular dichroism spectroscopy. Anal. Chem. 80: 9534-9541

Karnik R, Gu F, Basto P, Cannizzaro C, Dean L, Kyei-Manu W and Langer R 2008 Microfluidic platform for controlled synthesis of polymeric nanoparticles. Nano Lett. 8(9): 2906-2912

Kimling J, Maier M, Okenve B, Kotaidis V, Ballot H and Plech A 2006 Turkevich method for gold nanoparticle synthesis revisited. J. Phys. Chem. B 110(32): 1570015707

Knight J B, Vishwanath A, Brody J P and Austin R H 1998 Hydrodynamic Focusing on a Silicon Chip: Mixing Nanoliters in Microseconds. Phys. Rev. Lett. 80: 3863

Lee S, Kim D, Lee S and T Kwon T 2006 A split and recombination micromixer fabricated in a PDMS three-dimensional structure. J. Micromech. Microeng. 16: 1067

Lee W B, Weng C H, Cheng F Y, Yeh C S, Lei H Y and Lee G B 2009 Biomedical microdevices synthesis of iron oxide nanoparticles using a microfluidic system. Biomed. Microdevices 11: 161-171

Lee C Y, Chang C L, Wang Y N and Fu L M 2011 Microfluidic mixing: A review. Int. J. Mol. Sci. 12(5): 3263-3287

Li S, Xu J, Wang Y and Luo G 2008 Controllable preparation of nanoparticles by drops and plugs flow in a microchannel device. Langmuir 24(8): 41949

Lin X Z, Terepka A D and Yang H 2004 Synthesis of silver nanoparticles in a continuous flow tubular microreactor. Nano Lett. 4(11): 2227-2232

Liu R H, Stremler M A, Sharp K V, Olsen M G, Santiago J G, Adrian R J, Aref H and Beebe D J 2000 Passive mixing in a three-dimensional serpentine microchannel. J. Microelectromech. Syst. 9: 90-197

Markovich G, Left D V, Chung S W, Soyez H M, Dunn B and Heath J R 1997 Parallel fabrication and single-electron charging of devices based on ordered, two dimensional phases of organically functionalized metal nanocrystals. Appl. Phys. Lett. 70: 3107-3109

Matsui I 2006 Preparation of magnetic nanoparticles by pulsed plasma chemical vapor synthesis. $J$. Nanopart. Res. 8: 429-443

Ottino J M and Wiggins S 2004 Introduction: mixing in microfluidics. Philos. Trans. Ser. A Math. Phys. Eng. Sci. 362(1818): 923-935

Panyam J and Labhasetwar V 2003 Biodegradable nanoparticles for drug and gene delivery to cells and tissue. Adv. Drug Deliver Rev. 55: 329347

Peng G, Tisch U, Adams O, Hakim M, Shehada N, Broza Y Y, Billan S, Abdah-Bortnyak R, Kuten A and Haick H 2009 Diagnosing lung cancer in exhaled breath using gold nanoparticles. Nat. Nanotechnol. 4: $669-673$ 
Perriere J, Boulmer-Leborgne C, Benzerga R and Tricot S 2007 Nanoparticle formation by femtosecond laser ablation. J. Phys. D: Appl. Phys. 40: 7069-7076

Posner J and Santiago J J 2006 Convective instability of electrokinetic flows in a cross-shaped microchannel. J. Fluid Mech. 555: 1-42

Rhee M, Valencia P M, Rodriguez M I, Langer R, Farokhzad O C and Karnik R 2011 Synthesis of size-tunable polymeric nanoparticles enabled by 3D hydrodynamic flow focusing in single-layer microchannels. Adv. Mater. 23(12): 7983

Rudin T, Wegner K and Pratsinis S E 2011 Uniform nanoparticles by flame assisted spray pyrolysis (FASP) of low cost precursors. J. Nanopart. Res. 13: 2715-2725

Satyanarayana S, Karnik R N, Majumdar A and Mems A M 2005 Stamp-and-stick room-temperature bonding technique for microdevices. J. Microelectromech. Syst. 14(2): 392-399

Schmid G 2008 Generation and electrical contacting of gold quantum dots. Coll. Polym. Sci. 286: 1029

Schmid G and Simon U 2005 Gold nanoparticles: Assembly and electrical properties in 13 dimensions. Chem. Commun. 6: 697710

Shipway A N, Katz E and Willner I 2000 Nanoparticle arrays on surfaces for electronic, optical, and sensor applications. Chem. Phys. Chem. 1: 18-52

Sivaraman S K, Kumar S and Santhanam V 2010 Room-temperature synthesis of gold nanoparticles-Sizecontrol by slow addition. Gold Bull. 43(4): 275286

Somorjai G A and Park J Y 2008 Colloid science of metal nanoparticle catalysts in 2D and 3D Structures. Challenges of nucleation, growth, composition, particle shape, size control and their influence on activity and selectivity. Topics Catalysis 49: 126-135

Song Y, Kumar C S and Hormes J 2004 Synthesis of palladium nanoparticles using a continuous flow polymeric micro-reactor. J Nanosci. Nanotechnol. 4: 788793

Stroock A D, Dertinger S K W, Ajdari A, Mezic I, Stone H A and Whitesides G M 2002 Chaotic mixer for microchannels. Nature 295: 647-651

Teoh W Y, Amal R and Madler L 2010 Flame spray pyrolysis: An enabling technology for nanoparticles design and fabrication. Nanoscale 2: 1324-1347

Turkevich J, Stevenson P C and Hillier J 1951 A study of the nucleation and growth processes in the synthesis of colloidal gold. Discuss. Faraday. Soc. 11: 5575

Verma M K S and Kumaran V 2012 A dynamical instability due to fluid-wall coupling lowers the transition Reynolds number in the flow through a flexible tube. J. Fluid Mech. 705: 322-347

Verma M K S and Kumaran V 2013 A multifold reduction in the transition Reynolds number, and ultra-fast mixing, in a micro-channel due to a dynamical instability induced by a soft wall. J. Fluid Mech. 727: 407-455

Verma M K S, Ganneboyina S R, Vinayak R and Ghatak A 2008 Three-dimensional multihelical microfluidic mixers for rapid mixing of liquids. Langmuir 24: 22482251

Wagner J, Kirner T, Mayer G, Albert J and Kohler J 2004 Generation of metal nanoparticles in a microchannel reactor. Chem. Eng. J. 101(13): 251-260

Wang W, Yang C, Cui X Q, Bao Q L and Li C M 2010 Droplet microfluidic preparation of au nanoparticles-coated chitosan microbeads for flow-through surface-enhanced Raman scattering detection. Microfluidics Nanofluidics 9(6): 1175-1183 